\title{
1 Performance of A Compact Multi-crystal High-purity 2 Germanium Detector Array for Measuring Coincident 3 Gamma-ray Emissions
}

4 Chris Howard ${ }^{1,2}$, Stephen Daigle ${ }^{1,2}$, Matt Buckner ${ }^{1,2}$, Luke E. Erikson ${ }^{3}$, Robert C. Runkle ${ }^{3}$, Sean C. Stave ${ }^{3}$, Arthur

5 E. Champagne ${ }^{1,2}$, Andrew Cooper ${ }^{1,2}$, Lori Downen ${ }^{1,2}$, Brian D. Glasgow ${ }^{3}$, Keegan Kelly ${ }^{1,2}$, Anne Sallaska ${ }^{1,2}$

${ }^{1}$ University of North Carolina at Chapel Hill, Chapel Hill, NC 27599, USA

${ }^{2}$ Triangle Universities Nuclear Laboratory, Durham, NC 27708, USA

${ }^{3}$ Pacific Northwest National Laboratory, Richland, WA 99352, USA

Abstract: The Multi-sensor Airborne Radiation Survey (MARS) detector is a 14-crystal array of high-purity germanium (HPGe) detectors housed in a single cryostat. The array was used to measure the astrophysical Sfactor for the ${ }^{14} \mathrm{~N}(\mathrm{p}, \gamma)^{15} \mathrm{O}^{*}$ reaction for several transition energies at an effective center-of-mass energy of $163 \mathrm{keV}$. Owing to the granular nature of the MARS detector, the effect of gamma-ray summing was greatly reduced in comparison to past experiments which utilized large, single-crystal detectors. The new S-factor values agree within their uncertainties with the past measurements. Details of the analysis and detector performance are presented.

Keywords: HPGe array; high purity germanium; S-factor; ${ }^{14} \mathrm{~N}(\mathrm{p}, \gamma)^{15} \mathrm{O}^{*}$; summing corrections; granular

\section{Introduction}

This manuscript presents results from an initial measurement campaign using a custom-built, selfcontained compact array of high-purity germanium (HPGe) crystals called the Multi-sensor Airborne Radiation Survey (MARS) detector [1]. This detector system was deployed to the Laboratory for Experimental Nuclear Astrophysics (LENA) [2] for a proof-of-principle demonstration of the advantages offered by customized gamma-ray spectrometers for measuring nuclear cross-sections in acceleratorbased experiments. Using the ${ }^{14} \mathrm{~N}(p, \gamma)^{15} \mathrm{O}^{*}$ reaction as an example, we quantified the performance gains achievable in terms of reducing systematic errors associated with measuring radiation capture with coincidence gamma-ray emissions. The decay of the excited ${ }^{15} \mathrm{O}$ nucleus is shown in Figure 1 . Further, we explored the performance of various schema for selecting events that maximize reaction signatures in comparison to beam-induced and room-background signatures. 


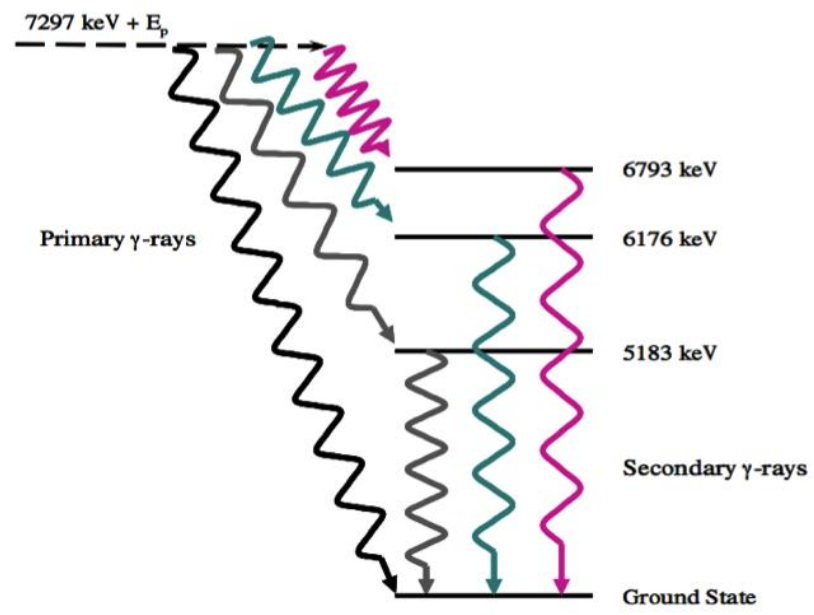

Figure 1: Decay scheme of ${ }^{15} \mathrm{O}$

A large HPGe detector made using a single crystal has a high absolute detection efficiency but will suffer from summing effects. Two gamma-rays emitted in a cascade can be so close in time as to appear as a single gamma-ray if both interact in the detector. Methods exist for mitigating the impact of summing effects (see [3]), but corrections based on these methods are uncertain at a level significant compared to other sources of systematic uncertainty in the extraction of the astrophysical S-factor. Summing will also affect the acceptable event rates so that the summing correction is kept relatively small, again, to minimize systematic errors. To keep a summing correction relatively small, additional distance between a source and detector must be allowed, reducing the probability of two (isotropically) emitted gamma-rays being simultaneously detected. This additional distance lowers the geometric efficiency for measuring decays in the sample and thus is an undesirable solution to the problem of summing effects. When multiple, small HPGe crystals are used, the probability of two coincident gamma rays interacting in the same crystal is decreased. By operating an array of individual crystals, the efficiency of a larger single crystal can be achieved but with much-reduced summing effects.

Several recent results for the cross-section ${ }^{14} \mathrm{~N}(\mathrm{p}, \gamma)^{15} \mathrm{O}^{*}$ at astrophysically-relevant energies exist in the literature [4-7]. These experiments were performed using a variety of detectors ranging from wellcharacterized, large, single-crystal HPGe detectors, to a small array of HPGe crystals to a large BGO array with nearly $4 \pi$ coverage. Gamma-ray emissions from ${ }^{14} \mathrm{~N}(\mathrm{p}, \gamma)^{15} \mathrm{O}^{*}$ exemplify the signatures commonly found in low-energy nuclear reactions where the density of nuclear excitation levels is modest. However, the reaction mechanism features a complicated interplay of resonances (including a subthreshold resonance at $-506 \mathrm{keV}$ ), direct capture and interference amongst the resonances, and between resonances and direct capture. In particular, the ground-state transition exhibits a pronounced interference dip arising from the interference of the tails of the subthreshold resonance with higherlying resonances. Lemut et al. employed a large, high efficiency bismuth germanate crystal to measure the total cross section down to a center-of-mass energy $\left(E_{c m}\right)$ of $70 \mathrm{keV}[6]$. However, these results could not be extrapolated to the lower energies of interest. Such an extrapolation requires precision measurements of the individual $\gamma$-ray transitions that follow proton capture. 


\section{Experimental Setup}

In this proof-of-concept experiment, the MARS detector was placed as close to the target position as possible. The goal was to acquire enough statistics to reach uncertainties that were similar in size to those in the data in Reference [7]. To make this measurement we were only able to detect the photons from the secondary transitions (i.e. transitions in the residual excited ${ }^{15} \mathrm{O}$ nucleus), due to the relatively high amount of room background. The primaries for each transition (i.e. gamma-rays emitted as the ${ }^{14} \mathrm{~N}+\mathrm{p}$ system de-excites to an excited ${ }^{15} \mathrm{O}$ nucleus) had an energy of less than $2300 \mathrm{keV}$, and thus were overwhelmed by the $U$ and Th decay daughter gamma rays which extend up to $2600 \mathrm{keV}$. Technically, the transition directly to the ground state of ${ }^{15} \mathrm{O}$ is also a primary and has a large enough energy to be above the low energy background but the branching ratio is very small making this a difficult transition to observe. Since the detector was only available for a limited amount of time (approximately 10 days), a beam energy of $E_{l a b}=180 \mathrm{keV}$ was chosen as a compromise between the need to measure at low energies and the necessity of acquiring significant statistics in the time allotted. Assuming a thin dead layer on the surface of the target of $0.5 \mathrm{keV}$, this gave an effective center-of-mass (CM) energy of $E_{\text {eff }}=163 \mathrm{keV}$. Yield curves were acquired at the $E_{\mathrm{lab}}=278 \mathrm{keV}\left(E_{\mathrm{cm}}=259 \mathrm{keV}\right)$ resonance before and after each low energy run.

Two different LENA accelerators were used for this experiment [2]. The 1-MV JN Van de Graff tandem accelerator was used for the yield curve measurements near the $278 \mathrm{keV}$ resonance, for which only relatively small amounts of beam current were required $(\sim 30 \mu \mathrm{A})$. The lower-energy, off-resonance measurements employed the electron-cyclotron-resonance (ECR) accelerator because of the much larger current it could provide (500 to $1000 \mu \mathrm{A}$ ). The current incident on the target was measured directly using an Ortec model 439 digital current integrator. Synchronization of the total integrated current and the MARS data acquisition system was maintained manually; the MARS data acquisition system would be started then the beam stop would be removed immediately afterward to deliver beam to the target. The process was reversed at the end of a run, which would last between 5 and 30 minutes. The systematic error introduced was minimal (estimated at $2 \%$ ) as no signal counts were lost and, for several additional seconds, only the background would be counted. The background near each peak was fit and removed during analysis, thus minimizing even this small error.

\subsection{Targets}

\subsubsection{Target Fabrication}

The nitrogen targets used in the MARS experiment consisted of $0.35 \pm 0.05 \mathrm{~mm}$ thick tantalum foils (99.95\% metals-basis purity) implanted with $\mathrm{N}_{2}{ }^{+}$ions. The $38 \mathrm{~mm} \times 38 \mathrm{~mm}$ square tantalum backings were wet-etched and resistively heated to remove surface contaminants. The wet-etching acid solution was made up of five parts $95 \%$ sulfuric acid $\left(\mathrm{H}_{2} \mathrm{SO}_{4}\right)$, two parts $70 \%$ nitric acid $\left(\mathrm{HNO}_{3}\right)$, and two parts $50 \%$ hydrofluoric acid (HF) [8]. The etched backings were rinsed in 200-proof ethanol and resistively heated inside a high-vacuum ( $10^{-7}$ Torr), oil-free evaporator system at LENA. The target backings were stored in an evacuated polycarbonate target box to inhibit the formation of an oxide layer on the surface of the tantalum. 
The prepared tantalum backings were implanted with nitrogen using an Eaton NV-3206 ion implanter at the University of North Carolina. The $10-k e V$-thick targets measured at the $E_{r}^{\text {lab }}=278 \mathrm{keV}$ resonance were produced using a $40-\mathrm{keV} \mathrm{N}_{2}{ }^{+}$ion beam with an incident dose of $30 \mu \mathrm{g} / \mathrm{cm}^{2}$. The $\mathrm{N}_{2}^{+}$ beam was raster-scanned across the surface of the tantalum backing, and a beam scanner was utilized to monitor the beam profile during implantation. Typical implantation time to reach saturation was about five hours with an average beam current of 30-40 $\mu \mathrm{A}$. The target chamber pressure was maintained below $5 \times 10^{-7}$ Torr; a liquid-nitrogen-cooled copper shroud positioned before the target prevented carbon and other contaminants from plating onto the surface of the tantalum backing.

\subsubsection{Target Composition}

The target composition stoichiometry was determined through Rutherford Backscattering Spectrometry (RBS) performed at the Triangle Universities Nuclear Laboratory (TUNL) tandem lab. The backscattered alpha particles were detected at 160 degrees. The RBS measurement utilized a LabVIEWcontrolled, semi-automatic target system [9]. A $3 \mathrm{~mm} \times 3 \mathrm{~mm}$ collimated ${ }^{4} \mathrm{He}^{+}$ion beam enabled precise measurements on bare tantalum ( $\mathrm{Ta}$ ) and nitrogen-implanted tantalum $\left(\mathrm{Ta}_{\mathrm{m}} \mathrm{N}_{\mathrm{n}}\right.$ ) regions on the targets. The backscattering yield in the Ta and $\operatorname{Ta}_{m} N_{n}$ samples was extracted from the plateau heights $\left(H^{T a}\right.$ and $H_{T a}^{T a_{m} N_{n}}$ ) in the Ta silicon detector energy spectra shown in Figure 2. The plateau is very narrow for targets of this thickness and the slope is indicative of tailing of the implanted $\mathrm{N}$ into the Ta backing. The stoichiometry was calculated using

$$
\frac{n}{m}=\frac{n_{\mathrm{N}}}{n_{\mathrm{Ta}}}=\frac{\epsilon_{\mathrm{Ta}}}{\epsilon_{\mathrm{N}}}\left(\frac{H_{\mathrm{Ta}}}{H_{\mathrm{Ta}}^{\mathrm{Ta} m} \mathrm{M}_{\eta}}-1\right)(1)
$$

where $\epsilon_{\mathrm{Ta}}$ and $\epsilon_{\mathrm{N}}$ are the stopping powers of $2.0 \mathrm{MeV}^{4} \mathrm{He}^{+}$ions in tantalum and nitrogen respectively. Equation 1 (from Ref. [10]) is a simplified version of Eqn. 18 from Ref. [11], appropriate for the case where a thin layer of a light element is mixed with a heavy substrate. It implicitly assumes that the stopping power is approximately constant over the width of the implanted layer. In the present case, the $\mathrm{Ta}_{\mathrm{m}} \mathrm{N}_{\mathrm{n}}$ layer is approximately $10-\mathrm{keV}$ for $278-\mathrm{keV}$ protons, which implies a thickness of approximately $6 \mathrm{keV}$ for 2-MeV alpha particles. This causes a negligible change in the stopping power. The stopping powers used in Equation 1 were calculated from the SRIM2013 computer application [12]. A total of seven targets were measured and a weighted average for targets used in the MARS experiment was $\mathrm{n} / \mathrm{m}$ $=1.5$ (1) (implying $\mathrm{Ta}_{2} \mathrm{~N}_{3}$ ), which is consistent with published values [7, 10]. The uncertainty includes the statistical uncertainty associated with the plateau and overall estimates of the uncertainties in the stopping powers. 


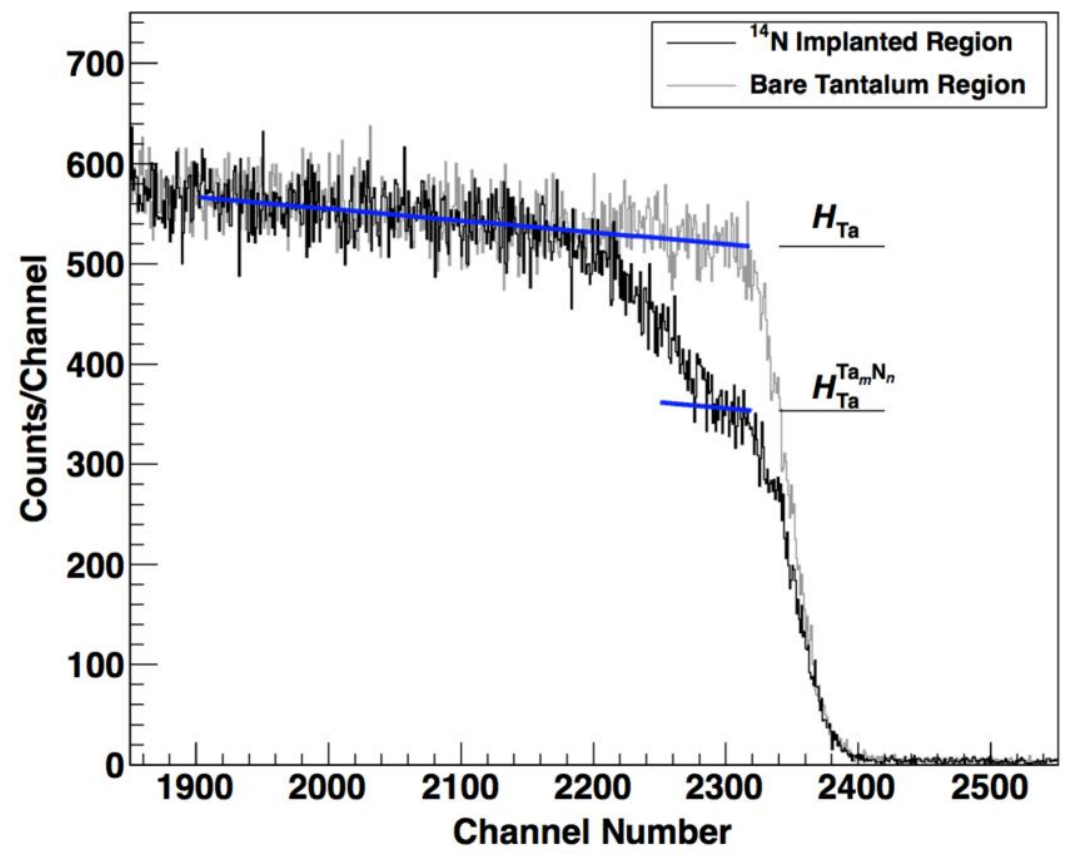

128

Figure 2. Silicon detector RBS energy spectra for bare tantalum and nitrogen implanted regions on a sample target prepared for the MARS experiment.

\subsubsection{Target Profiles}

Target profiles were recorded periodically throughout the MARS experiment to monitor the condition of each target. Yield curves of the $E_{l a b}=278 \mathrm{keV}$ resonance in ${ }^{14} \mathrm{~N}(p, \gamma){ }^{15} \mathrm{O}^{*}$ were measured using the JN Van de Graaff proton accelerator at LENA. Measured yield curves before and after 8.1 coulombs $(C)$ of proton beam are shown in Figure 3 and were obtained from the intensity of the primary transition to the $6.18 \mathrm{MeV}$ state in ${ }^{15} \mathrm{O}$. The target does thin after an accumulated charge of $8.1 \mathrm{C}$, however the yield (represented by the peak height in Figure 3 ) remains constant. 
Excitation Functions for ${ }^{14} \mathrm{~N}(\mathrm{p}$, ) with MARS: Target 3

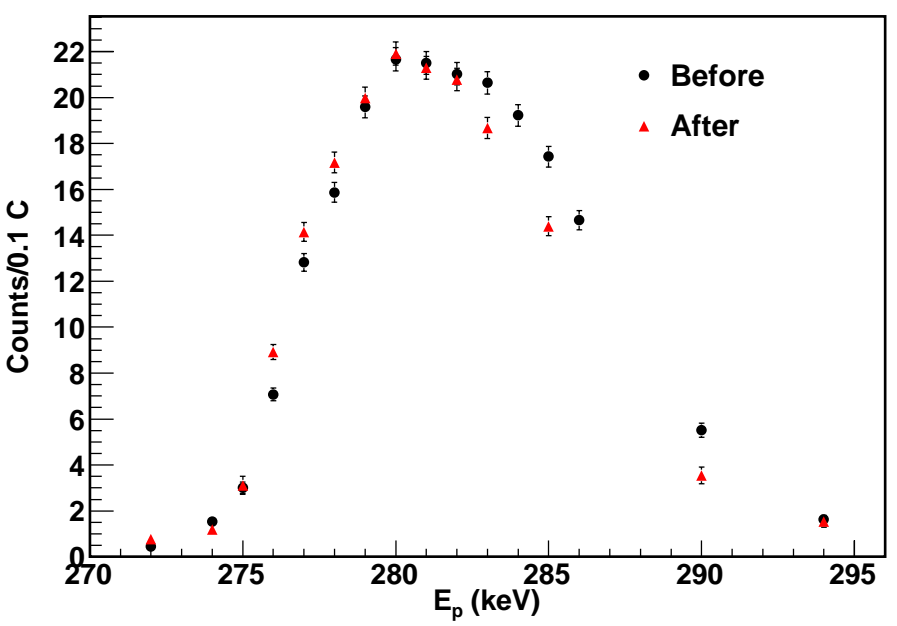

Figure 3. Measured yield curves of the $\mathrm{E}_{\mathrm{lab}}=278 \mathrm{keV}$ resonance before and after accumulating $8.1 \mathrm{C}$ of proton beam from the JN Van de Graaff accelerator during the MARS experiment. Note that the maximum yield occurs at $280 \mathrm{keV}$, which is a convolution of the energy spread of the proton beam and a thin dead layer on the surface of the target.

\subsection{Detection System}

Pacific Northwest National Laboratory developed a customized multi-element gamma-ray spectrometer for nuclear security missions [1]. This system, called the Multi-sensor Airborne Radiation Survey (MARS) detector, consists of 14 high-purity germanium (HPGe) crystals in a close-packed array contained within a single cryostat. However, for this experiment, only 12 of the HPGe crystals were operating. Each p-type coaxial crystal measures approximately $6.2 \mathrm{~cm}$ in diameter and $4.5 \mathrm{~cm}$ in length with a relative efficiency (RE) of $\sim 38 \%$. (Efficiency of HPGe crystals is typically measured relative to a 3" $\times 3 " \mathrm{Nal}(\mathrm{TI})$ crystal. See Ref. [13]). The crystals are arranged in two close-packed arrays each containing 7 crystals. One crystal is located in the center of each platter with the remaining 6 crystals in a ring around the central crystal (see Figure 4). The two platters are located next to each other separated by approximately $2 \mathrm{~cm}$. The detector was originally designed for use on aircraft, boats, and trucks for characterization and stand-off detection of various radioactive materials. The largest single HPGe crystals manufactured are typically up to $140 \%$ RE (some specialty research crystals have $>200 \%$ RE [14]), so multiple crystals are required to assemble higher efficiency systems. However, crystal cost does not scale linearly with size because of the difficulty in manufacturing the larger crystals. The $38 \%$ RE size was at the optimal balance between efficiency and cost. 


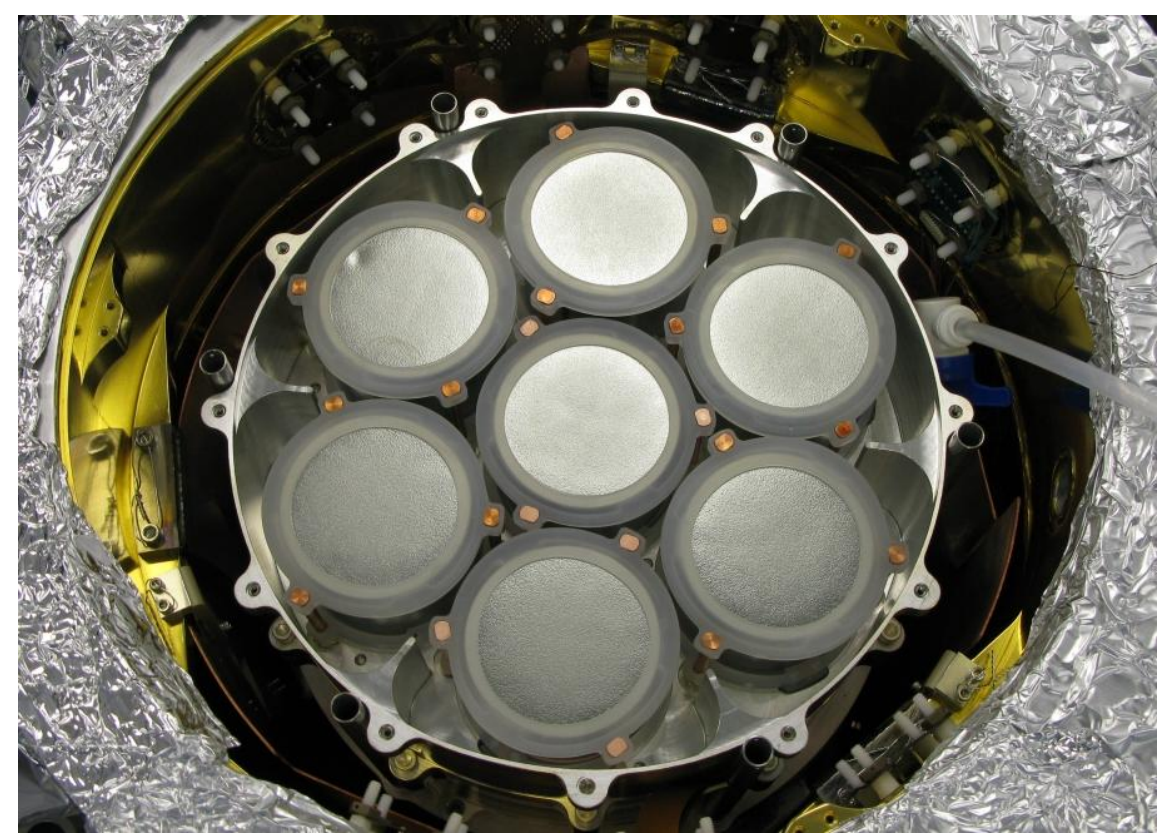

Figure 4. Photograph of the front MARS detector platter with the outer window and heat shields removed. The rear platter is not visible but is behind the front platter and rotated 30 degrees.

The first stage of amplification occurs very close to each HPGe crystal. The components are located close-by to minimize detector capacitance and thus reduce noise; the cold temperatures also reduce the noise in the field effect transistor. The signal from the cold components is sent to a preamplifier located on the outside of the cryostat. The preamp signal is then sent to a Pixie-4 waveform digitizer [15] made by $\mathrm{XIA}^{1}$. A more detailed explanation of the MARS electronics is given in Ref. [1]. The full waveform data are not recorded to disk but multiple trapezoidal filters are applied to the waveform onboard the digitizer card, yielding a pulse height and a time for each pulse. For the MARS array to act as a single crystal, coincident pulses must be summed together, returning counts to the full energy peak that otherwise would have been seen as two partial deposition events. This coincidence summation is performed offline and uses the energy calibration constants determined separately for each crystal. For this experiment, the fact that each crystal is read out independently is critical. Two gamma rays from a cascade are much more likely to be detected in two separate crystals owing to the granularity of MARS and distance from the target. The MARS electronics allow for each of these interactions to be observed instead of only the sum as would be the case in a large, single crystal.

The Pixie-4 digitizer operates at $75 \mathrm{MHz}$ (13.3 ns time steps) and has a field programmable gate array (FPGA), memory, and a digital signal processor onboard that together perform operations on the waveform. This imparts a great speed advantage by reducing the amount of data sent to the control computer. An output of a trapezoidal filter with a rise time and flat top both equal to 195 ns was used to trigger the digitizer and establish the time of the event. Then, a second filter with a rise time of 11.9 $\mu \mathrm{s}$ and a flat top of $1.28 \mu \mathrm{s}$ was used to determine the event pulse height. The trapezoidal filter settings had been optimized previously and have been sufficient to achieve individual crystal energy resolutions of 2 to $3 \mathrm{keV}$ full width at half maximum (FWHM) at $1333 \mathrm{keV}$. Typical event rates in the MARS detector are low enough that nanosecond timing resolution is not required. The criterion for a coincidence event 
is two samples falling within $1 \mu \mathrm{s}$ of each other, which translates to within 75 sampling times. This time is much longer than the charge collection time in the crystals and is not a limiting factor in the measurement. The system was operated using custom software built using libraries provided by XIA. At the event rates observed during the low-energy operation of the detector, the live time of the system was greater than $99.5 \%$ as determined by the internal clocks on the digitizer cards.

\subsection{Detector Geometry}

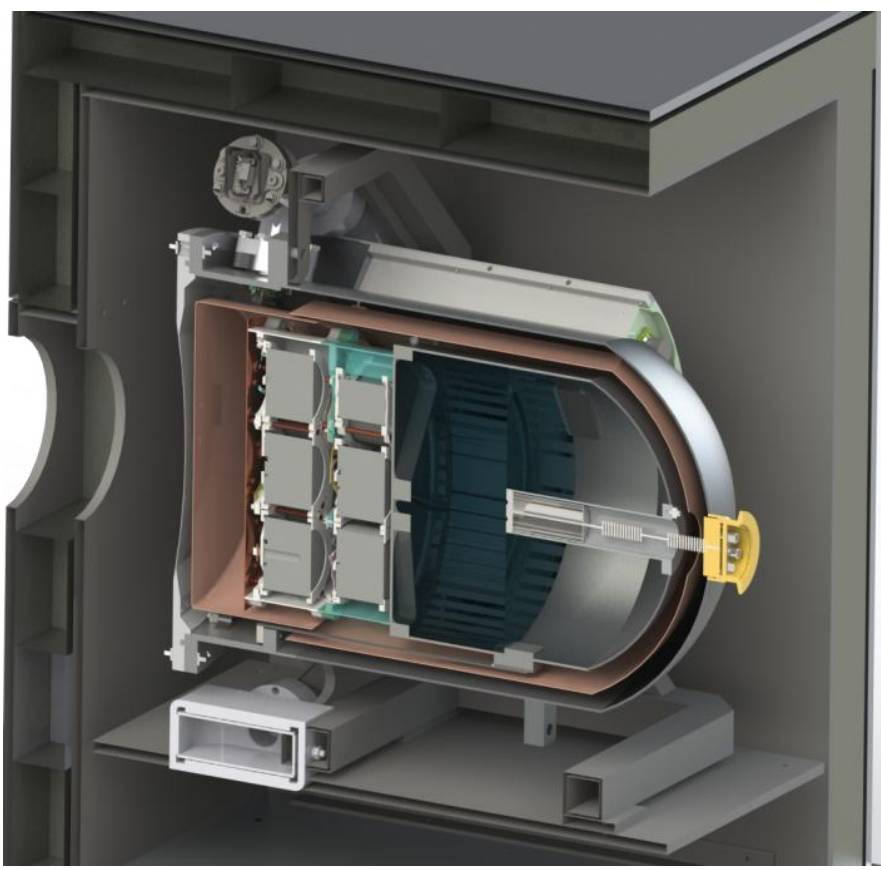

Figure 5. Side cutaway view of the MARS detector inside the 2" thick lead shield (downstream wall removed for clarity). The thin front window is on the left side of the figure and the LENA beamline extended through the front hole in the shield. The nitrogen fill port is on the right.

For this experiment, the MARS detector was oriented so that the thin front window was observing horizontally (see Figure 5). A 2-inch thick lead shield was built around the MARS detector to shield it from the natural background from natural sources and the copious amount of $x$-rays the LENA ECR source generates when operated at the energies used in these experiments. A small region near the front of the shield was left open to allow the beamline to extend into the shield. This lead shield also greatly decreased the natural background rate observed in MARS. Typical background rates observed in the lab are 400 to $500 \mathrm{~Hz}$. Inside the lead shield, the rate was $60 \mathrm{~Hz}$. This remaining natural background also provided a long-term gain stabilization signal.

The implanted targets were placed at the end of the beam line and were directly water-cooled and the outer window of the MARS detector was placed approximately $3 \mathrm{~mm}$ from the back of the target holder. The front face of the central crystal in MARS is approximately $6.35 \mathrm{~cm}$ from the window, producing a total distance to the target of $7.45 \mathrm{~cm}$. This estimated value was confirmed by performing two on-resonance measurements. The first measurement was performed at the standard detector 
212 location for 4 minutes ( $10 \mathrm{mC}$ of total charge). The detector was then translated a distance of $5 \mathrm{~cm}$ 213 farther from the target, and resonance data were acquired for 30 minutes ( $75 \mathrm{mC}$ of total charge). The

214 ratio of detector counts per charge at $6.176 \mathrm{MeV}$ was $1.92 \pm 0.10$. A GEANT4 simulation of the detector 215 with sources in both locations yielded a consistent ratio of $1.80 \pm 0.05$ at $6 \mathrm{MeV}$, thus confirming the 216 distance estimate.

\section{Detector Stability}

Many of the analysis techniques used for processing MARS spectroscopic data are sensitive to maintaining a stable energy calibration. Frequently, the signals from detector crystals are summed, but this approach can suffer from substantial peak widening caused by gain drift. Additionally, a problematic energy calibration tends to produce non-Gaussian peak shapes that can complicate peak fitting and counting. For these reasons, the stability and quality of the energy calibration were examined at least every time a new ECR measurement was conducted.

The MARS detector array was initially gain matched using energy calibrations from ${ }^{60} \mathrm{Co}$ and ${ }^{22} \mathrm{Na}$ button sources in addition to the $1461 \mathrm{keV}$ line from ${ }^{40} \mathrm{~K}$ and the $2614 \mathrm{keV}$ line from ${ }^{208} \mathrm{TI}\left({ }^{232} \mathrm{Th}\right.$ decay chain). However, gamma rays produced from the radiative capture experiment are clustered in the 5-7 $\mathrm{MeV}$ region which has no strong natural background lines. Fortunately, the frequent yield measurements on-resonance produced several strong peaks ranging from 5.154 MeV up through 6.793 MeV.

The yield measurements were conducted before and after the ECR based off-resonance measurements (see Figure 6 below). To minimize the effects of gain drift, the yield measurements immediately before ECR runs were used for energy calibration. An analysis of the resulting calibrations demonstrated that the crystals remained stable for the duration of the off-resonance runs that occurred between yield runs, but there were benefits to calibrating on every yield curve measured (see Figure 7 below). In this case, the $511 \mathrm{keV}$ (background), $1461 \mathrm{keV}\left({ }^{40} \mathrm{~K}\right), 5.183 \mathrm{MeV}$ (radiative capture) and 6.176 $\mathrm{MeV}$ (radiative capture) gamma rays were used for energy calibration for each crystal upon each yield 237 curve measurement. 


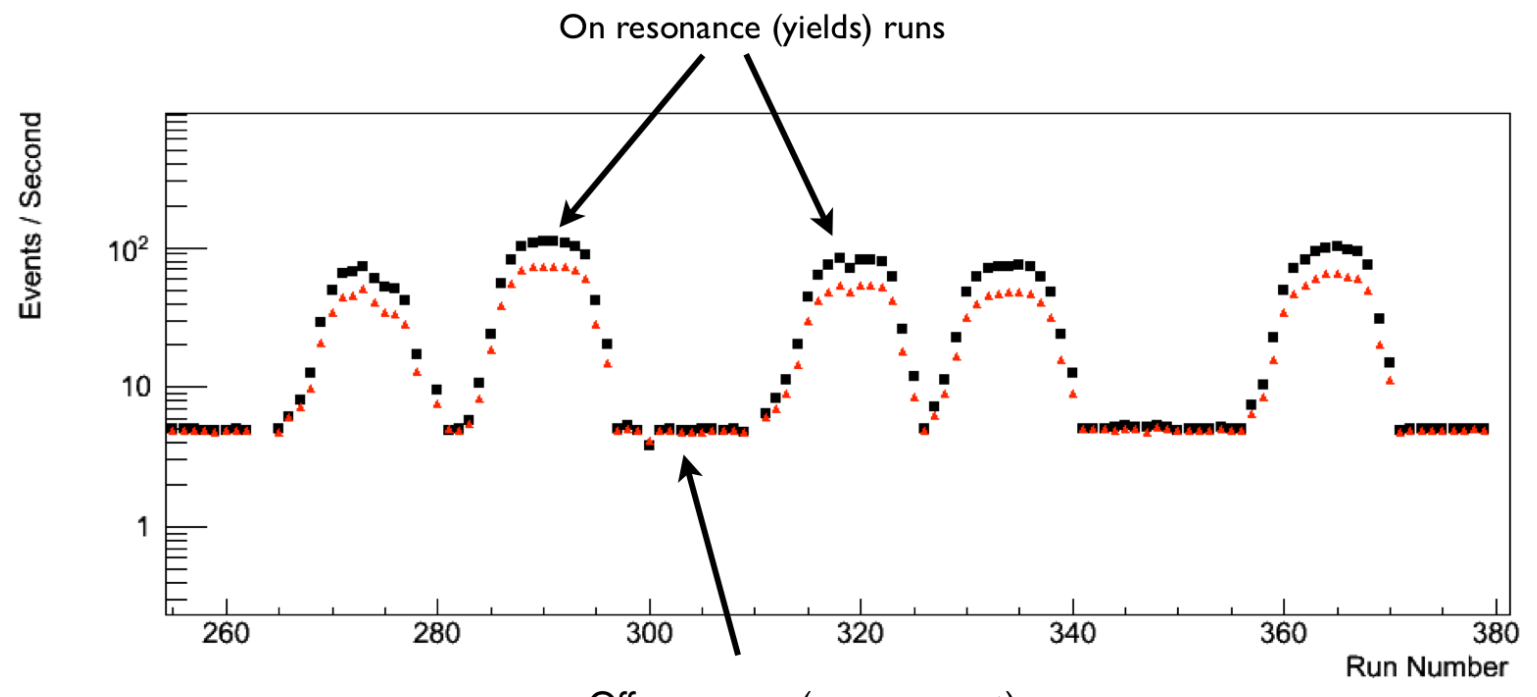

Off resonance (measurement) runs

241 Figure 6. Count rates are shown by run number where black squares indicate the rates in the center front crystal and red triangles are rates for a front outer crystal. Yield curves produce the high rates as the beam energy is adjusted in steps across the resonance energy. ECR-based off-resonance measurements have the lowest rates.

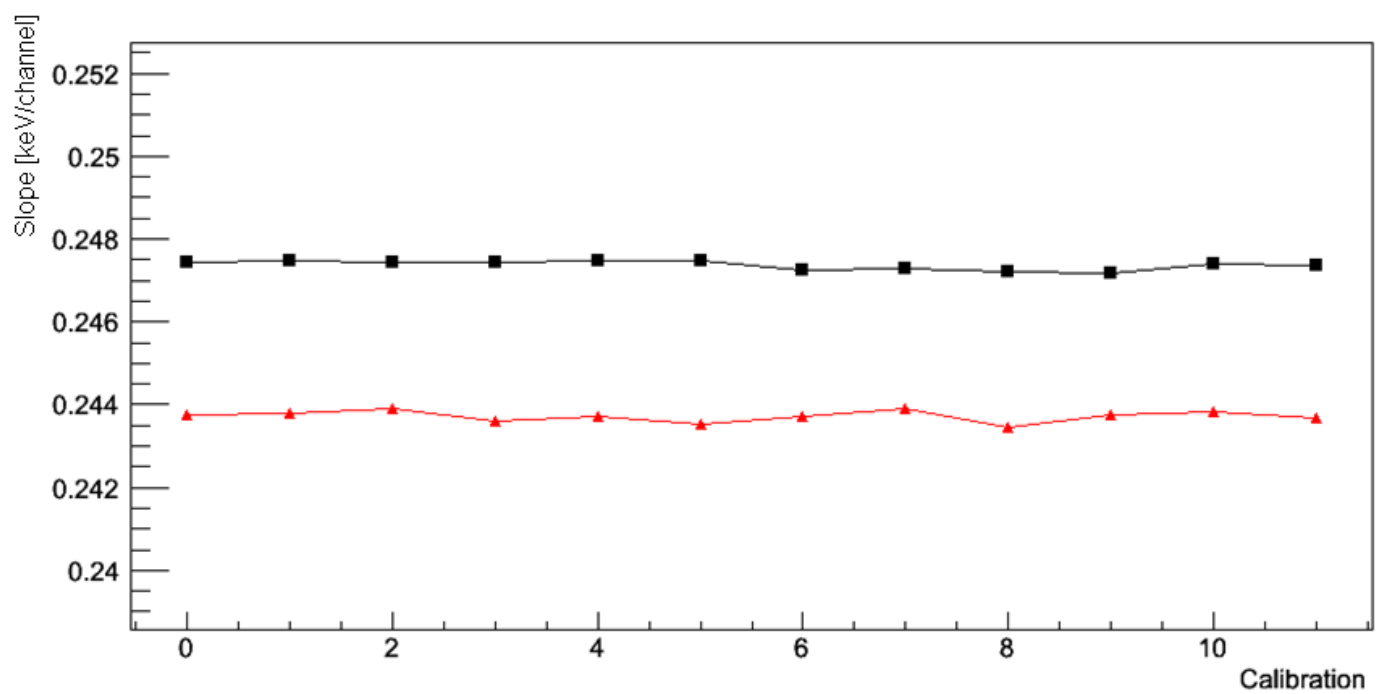

Figure 7. The slope $(B)$ of the energy calibration (for a Energy $=A+B *$ channel $+C *$ channel^2) energy calibration is shown for the center front crystal in MARS (black squares) and a front outer crystal (red triangles). The variation shown here is typical for those seen throughout the array for each calibration based on separate yield curves.

This approach provided a new energy calibration for the duration of an individual ECR measurement which would last approximately 5 hours between yield measurements. Figure 8 shows examples of the peak shapes and centroids for the $5.183 \mathrm{MeV}$ gamma that resulted from combining the array's gamma singles across different energy calibrations. Calibrations for each crystal taken at different times were 
applied in different ways and yet the peak shape and position remained constant. This similarity suggests that the ten separate energy calibrations (over time) on all twelve crystals can be used to combine singles events from ECR runs into spectra while maintaining good energy resolution.

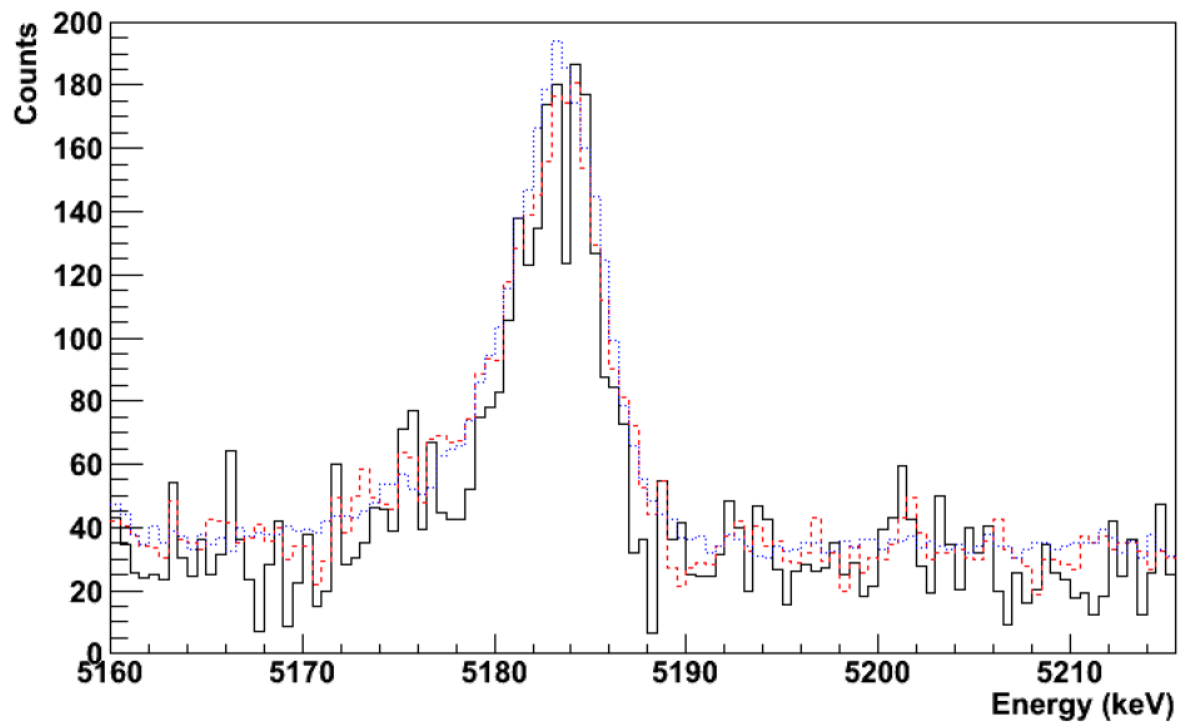

Figure 8. The $5.183 \mathrm{MeV}$ peak after energy calibration based on one yield curve run for the front center crystal (black line), the whole array for one energy calibration applied using a single yield curve run (red dashes), and the whole array with all energy calibrations applied to all yield measurements (blue dots). The similar positions and shapes indicate stability over time and consistency across crystals.

\section{Detector Efficiency}

As mentioned above, the measurement time with the MARS detector onsite at LENA was limited. Therefore, detailed detector characterization measurements were not performed. The on-resonance data were used to determine the efficiency of the MARS detector thus making the experiment a measurement relative to previous measurements of the resonance strength. The uncertainties from the previous measurements of the on-resonance strength will then be included in this measurement.

To calculate the efficiency, the net number of counts were extracted from the relevant peaks in the on-resonance runs (the spectra summed over all of the on-resonance runs is shown in Figure 9 with example peaks shown in Figure 10). The background under the peaks was assumed to be well approximated by the flat background near the peaks. The only approximation in the efficiency calculation is that the ground state efficiency is the same as it shifts with the beam energy-in this case $\approx 100 \mathrm{keV}$. This is acceptable because the efficiency curve is fairly flat at this high energy. Table 1 shows the number of counts at each energy. To produce these data, $0.231 \pm 0.006(2.5 \%) \mathrm{C}$ of charge was accumulated. 


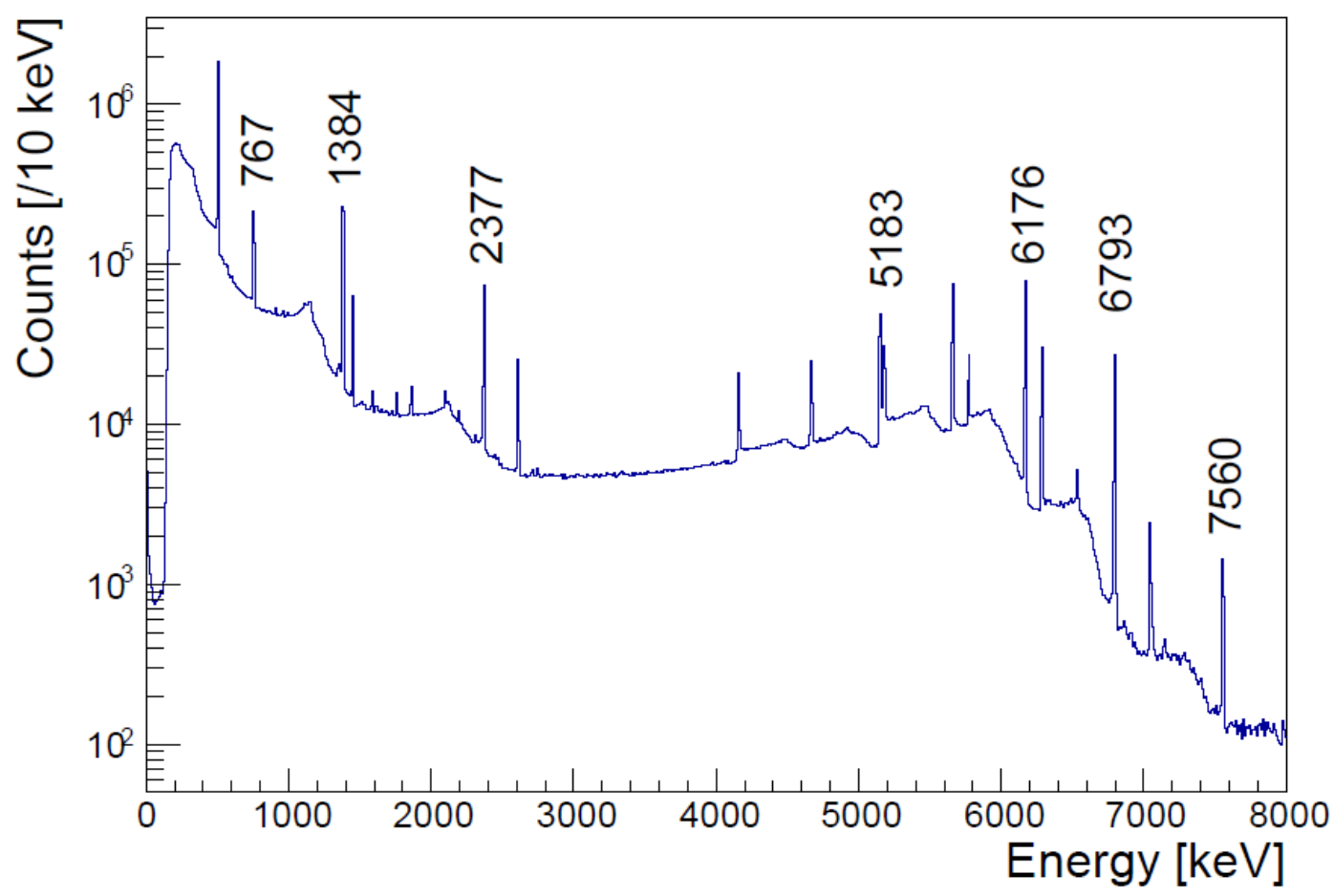

Figure 9. The spectrum resulting from the sum of all of the on-resonance data acquired with the MARS detector during the experiment run at LENA. Full-energy gamma-ray peaks of relevance have been annotated. Most of the smaller remaining peaks are first and second escape peaks related to the full-energy peaks. 


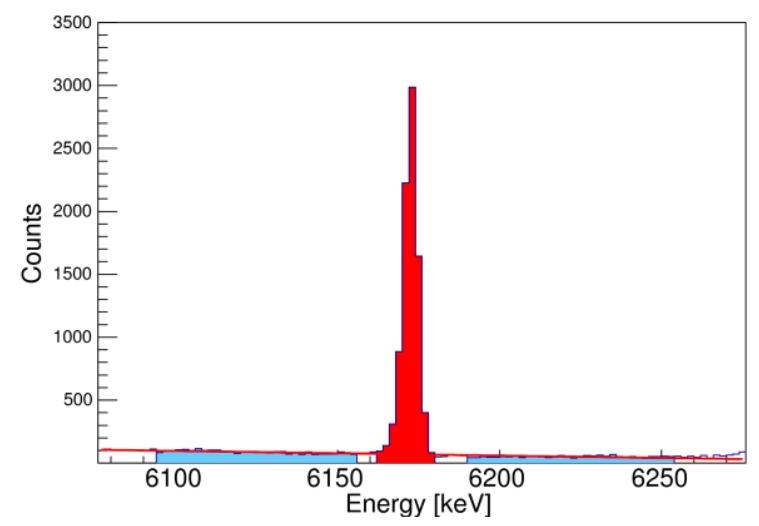

284
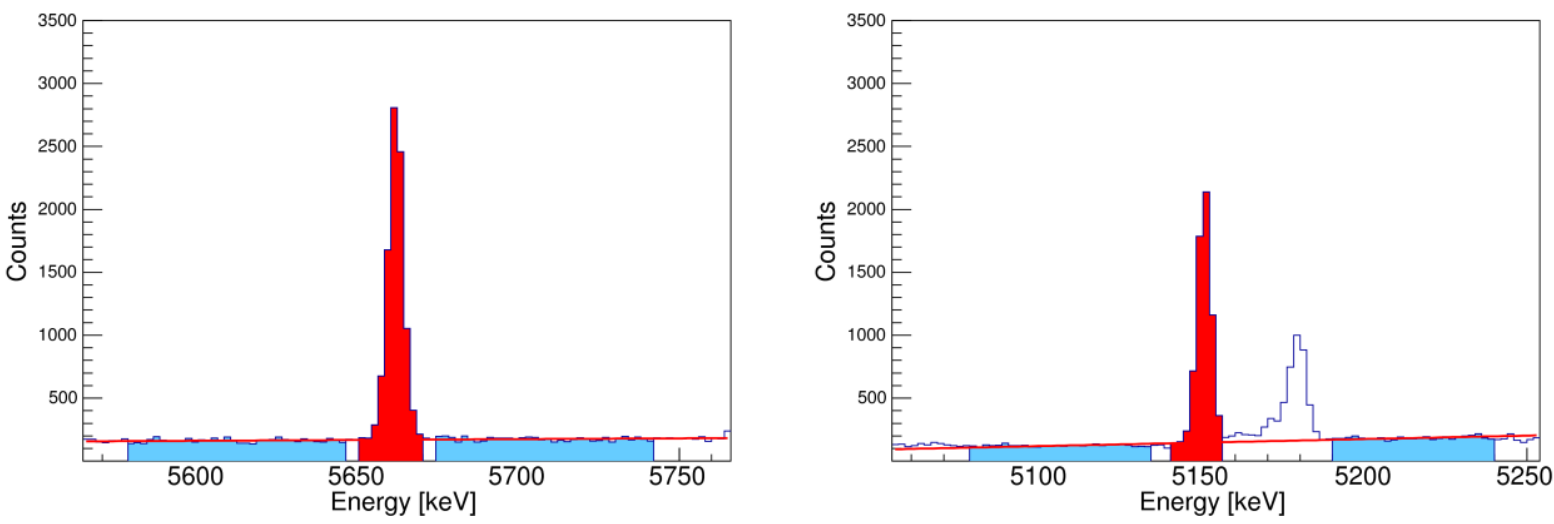

Figure 10. 6176 keV full-energy peak, first-escape peak, and second-escape peak

Table 1. Net counts at each energy (sum of full-energy + first-escape + second-escape peaks).

\begin{tabular}{|c|c|}
\hline Energy [keV] & Net Counts \\
\hline 5183 & $6584 \pm 104$ \\
6176 & $21917 \pm 161$ \\
6793 & $8052 \pm 102$ \\
GS & $689 \pm 35$ \\
\hline
\end{tabular}

The efficiency calculation began with Equation (4.123) from Nuclear Physics of Stars [16]:

$$
\omega \gamma=\frac{2 \epsilon_{\mathrm{eff}}}{\lambda_{\mathrm{dB}}^{2}} \frac{\mathrm{N}}{\mathrm{N}_{\mathrm{p}} \mathrm{B} \eta \mathrm{W}}[\mathrm{eV}]
$$

where $\omega \gamma$ is the resonance strength, $\epsilon_{\theta \mathrm{eff}}$ is the effective stopping power given by Equation (3), $\lambda_{\mathrm{dB}}^{2}$ is the deBroglie wavelength given by Equation (4), $\mathrm{N}_{\mathrm{p}}$ is the number of incident protons $\left(\mathrm{N}_{\mathrm{p}}=\mathrm{BCl} / 1.6 \times\right.$ $10^{-19} \mathrm{C}$ where $\mathrm{BCl}$ is total charge from the beam current integrator), $\mathrm{B}$ is the branching ratio given in Table 2, and the other parameters are in Table 3, W is the angular correlation which is assumed to be 1 , and $\eta$ is the efficiency. The efficiency at each energy is shown in Table 4. 


$$
\epsilon_{\mathrm{eff}}=\frac{M_{N}}{M_{N}+M_{p}}\left[\epsilon_{N}+\frac{n_{\mathrm{Ta}}}{n_{\mathrm{N}}} \epsilon_{\mathrm{Ta}}\right]\left[\mathrm{eVcm}^{2} / \text { atom }\right]_{(3)}
$$
It was already shown in Section 2.1.2 that $\frac{n_{\mathrm{Ta}}}{n_{\mathrm{N}}}=\frac{2}{3}$.

$$
\frac{\lambda_{d B}^{2}}{2}=\left(\frac{M_{p}+M_{n}}{M_{n}}\right) \frac{4.125 \times 10^{-18}}{M_{p} E_{b e a m}^{l a b}}\left[\mathrm{~cm}^{2}\right]_{(4)}
$$

302

Table 2: Branching ratios taken from literature values; Average of Runkle[7], LUNA[4-6]

\begin{tabular}{|cl|}
\hline Energy $[\mathrm{keV}]$ & Branching Ratio \\
\hline 5183 & $0.16875 \pm 0.002$ \\
6176 & $0.57975 \pm 0.002$ \\
6793 & $0.2285 \pm 0.002$ \\
GS & $0.015967 \pm 0.0013$ \\
\hline
\end{tabular}

Table 3: Parameters necessary to calculate the efficiency

\begin{tabular}{|cl|l|}
\hline Parameter & Value & Ref. \\
\hline$\omega / \mathrm{v}$ & $(13.1 \pm 0.6) \times 10^{-3} \mathrm{eV}$ & {$[17]$} \\
$\mathrm{M}_{\mathrm{p}}{ }^{+}$ & $1.00727638 \mathrm{amu}$ & {$[18]$} \\
$\mathrm{M}_{14 \mathrm{~N}}{ }^{+}$ & $14.0030740048 \mathrm{amu}$ & {$[19]$} \\
$\varepsilon_{\mathrm{Ta}}$ & $31.93 \times 10^{-15} \pm 5 \% \mathrm{eV} \mathrm{cm}{ }^{2} /$ atom & {$[12]$} \\
$\varepsilon^{14}{ }_{\mathrm{N}}$ & $10.71 \times 10^{-15} \pm 5 \% \mathrm{eV} \mathrm{cm} /$ atom & {$[12]$} \\
\hline $\mathrm{E}^{\text {lab }}{ }_{\text {beam }}$ & $280000 \mathrm{eV}$ & \\
\hline
\end{tabular}

Table 4: MARS absolute detection efficiency at each energy, as determined from on-resonance runs

\begin{tabular}{|c|c|}
\hline Energy [keV] & Efficiency \\
\hline 5183 & $0.00371 \pm 0.00025$ \\
6176 & $0.00360 \pm 0.00024$ \\
6793 & $0.00335 \pm 0.00023$ \\
GS & $0.00411 \pm 0.00048$ \\
\hline
\end{tabular}

\section{Effect of Granularity}

\section{$312 \quad 5.1$ Solid angle comparison}

313 The previous ${ }^{14} \mathrm{~N}(\mathrm{p}, \gamma)^{15} \mathrm{O}^{*}$ experiment at LENA used a $135 \%$ relative efficiency (RE) HPGe detector located

$3149 \mathrm{~mm}$ from the target [7]. The absolute photopeak efficiency of the detector had been determined

315 using calibrated sources and the sum-peak method of Reference [20]. As described above, the

316 photopeak efficiency of the MARS detector was determined using the known cross section of the 0.259

317 MeV resonance in the ${ }^{14} \mathrm{~N}(\mathrm{p}, \gamma)^{15} \mathrm{O}^{*}$ reaction along with the measured target thickness and beam current.

318 The results of both measurements are compared in Table 5. These results show that despite the 
distance to the front face of the MARS detector being more than 7 times farther away, the absolute photopeak efficiency decreased by less than a factor of 4 . These results are consistent with the large solid angles of the detectors relative to the distance from the target. In this case, the solid angle is expected to follow the form $2 \pi(1-\cos \theta)$ where $\theta$ is the angle corresponding to atan $(r / d)$ where $r$ is the radius of the detector and $d$ is the distance. For small angles, the equation can be reduced to $\pi r^{2} / d^{2}$ which exhibits the familiar variation with inverse distance squared. Using the full equation, a detector radius of $4.5 \mathrm{~cm}$, a near position of $9 \mathrm{~mm}$ and a far position of $74.5 \mathrm{~mm}$, the expected decrease in solid angle is a factor of 5.58. However, the front platter of MARS had 6 active crystals each of which is $38 \%$ RE for a total RE of $228 \%$. This leads to an expected efficiency ratio of 3.48 , based on geometry and detector size alone, which is in good agreement with the measurement results shown in Table 5 . It should also be noted that the efficiency decreases with increasing gamma-ray energy for both detectors as would be expected given the energy dependence of the attenuation coefficient of germanium at these energies.

Table 5. Comparison of absolute photopeak efficiencies for the $135 \%$ HPGe $9 \pm 1 \mathrm{~mm}$ from the target and the MARS detector at $7.45 \mathrm{~cm}$ from the target.

\begin{tabular}{|l|l|l|l|}
\hline Energy (keV) & $\begin{array}{l}\text { Absolute photopeak } \\
\text { efficiency } \\
135 \% \text { HPGe }\left(10^{-3}\right)\end{array}$ & $\begin{array}{l}\text { Absolute photopeak } \\
\text { efficiency } \\
\text { MARS }\left(10^{-3}\right)\end{array}$ & $\begin{array}{l}\text { Ratio } \\
135 \% / \text { MARS }\end{array}$ \\
\hline 5183 & $14.5 \pm 0.7$ & $3.71 \pm 0.3$ & $3.91 \pm 0.33$ \\
\hline 6176 & $12.5 \pm 0.6$ & $3.60 \pm 0.2$ & $3.46 \pm 0.29$ \\
\hline 6793 & $11.5 \pm 0.6$ & $3.35 \pm 0.2$ & $3.42 \pm 0.29$ \\
\hline
\end{tabular}

\subsection{Effect of singles vs. coincidence vs, anti-coincidence}

The MARS data were collected in "list-mode" for each crystal meaning that the energy and time of each event was recorded for each crystal. In the offline analysis, events that occurred within $1 \mu \mathrm{s}$ of each other could either be summed or vetoed depending upon which crystals were hit in the event. Previous work [1] has demonstrated the increase in efficiency in the MARS detector that can be gained by summing coincident events. However, in this analysis, it is critical to avoid the effects of summing. A study of the different ways of treating the coincidence data was performed on the counts collected in the $6793 \mathrm{keV}$ peak for $79.49 \mathrm{C}$ of total charge. The results are summarized in Table 6 . The most counts were acquired with the smallest statistical uncertainty using the add-back method (calibrated energies from coincident events were summed together) but the systematic uncertainty of the sum-correction causes this method to have larger total uncertainty than other methods. Summing all the crystals together yielded the next largest number of counts but the elevated background led to a larger net uncertainty. Next, a multiplicity cut of 1 was required where no other crystal had an event within $1 \mu \mathrm{s}$. This helped to reduce the background and also the uncertainty. Next, only events in the front layer of crystals were accepted. The relatively small decrease in the total number of counts shows that the majority of events are in the front layer of crystals, which would be expected (the front layer is closer and has a larger solid angle than the back layer plus the back layer is partially shielded by the front layer). To further reduce the background in the front layer, events were vetoed if there was a coincident event in the back layer of crystals. This marginally reduced the counts but lowered the background further. 
The statistics in this study are small but the systematic trends agree with the expected behavior. The most stringent cuts lead to a reduced background and smaller uncertainties. They also avoid the systematic effects that come from the add-back technique. The all-crystals front (veto rear) condition was used for all of the data analysis in this report.

Table 6. Comparison of different analysis techniques using the counts in the $6793 \mathrm{keV}$ peak. See text for details. The sum-correction is method is described in the following section but is included for comparison.

\begin{tabular}{|l|l|l|l|}
\hline Cut & Number of Counts & Uncertainty & \% error \\
\hline Add-back (not sum-corrected) & 116 & 24 & 20.69 \\
\hline Add-back (with sum-correction) & 106 & 31 & 29.25 \\
\hline All-crystals & 82 & 24 & 29.27 \\
\hline All-crystals (mult==1) & 75 & 19 & 25.33 \\
\hline All-crystals front & 71 & 19 & 26.76 \\
\hline All-crystals front (veto rear) & 70 & 17 & 24.29 \\
\hline
\end{tabular}

\subsection{Summing corrections for LENA vS. MARS}

\subsubsection{Trade-offs between summing and efficiency}

Summing corrections are necessary when there is a significant chance that $\gamma$ rays from a multi- $\gamma$-ray cascade will interact in the same detector at the same time yielding the same measured energy as the transition that avoided the intermediate level. As described above, the addition of systematic uncertainties can make summing corrections unattractive.

To determine the summing correction, $S C$, in the data, the add-back spectrum was compared to the individual spectrum and the ratio is the summing correction:

$$
S C=\frac{N_{\text {individual }}}{N_{\text {add-back }}}
$$

where $N_{\text {individual }}$ is the net number of counts in an individual crystal and $N_{\text {add-back }}$ is the net number of counts after summing coincident crystals.

The corrected number of counts is then given by

$$
N_{\text {corr }}=N_{\text {add-back }} S C
$$

Propagating the uncertainty leads to

$$
\delta N_{\text {corr }}=N_{\text {corr }} \sqrt{\left(\frac{\delta N_{a d d-b a c k}}{N_{\text {add-back }}}\right)^{2}+\left(\frac{\delta S C}{S C}\right)^{2}}
$$


where the $\delta$ prefix indicates the statistical uncertainty in the quantity.

The 6176 and $6793 \mathrm{keV}$ peaks were used in the analysis since those peaks had the highest statistics. Further, this analysis was performed on the resonance data as well as the off-resonance data. Table 7 contains the results of the analysis and shows that summing corrections are indeed large if MARS is treated as a single piece of germanium. While summing leads to an increase in efficiency, there is an associated increase in the overall uncertainty because of the required summing correction.

Table 7. Summing corrections for off-resonance and on-resonance data.

\begin{tabular}{|l|l|l|}
\hline Energy (keV) & Summing Corrections & Summing Corrections \\
& Off-resonance & On-resonance \\
\hline 6176 & $0.66 \pm 0.21$ & $0.79 \pm 0.01$ \\
\hline 6793 & $0.91 \pm 0.19$ & $0.79 \pm 0.01$ \\
\hline
\end{tabular}

The previous ${ }^{14} \mathrm{~N}(\mathrm{p}, \gamma)^{15} \mathrm{O}^{*}$ experiment at LENA with which this experiment is being compared had summing corrections of about $80 \%$ [7] which is about the same size as MARS would have if used as a single crystal. While the previous experiment used a single crystal, these larger summing corrections can be avoided if MARS is treated as separate HPGe detectors. However, each crystal is still susceptible to summing within it that cannot be eliminated with cuts. The size of the summing effect was measured using a ${ }^{60}$ Co source in the same location as the beam line target. Then the counts in the 1173 and 1333 $\mathrm{keV}$ peaks were compared with the counts in the $2506 \mathrm{keV}$ sum peak for the central crystal, 1 of the edge crystals and for the entire front layer of crystals. The results are summarized in Table 8 . The central crystal was the closest to the source and presented the largest solid angle and so was most susceptible to summing. However, the fraction of total events which underwent summing is relatively small at $0.42 \%$. Edge crystals were farther from the source and also presented a smaller solid angle, leading to a summing effect of $0.21 \%$. As a whole, the entire front layer of crystals would be expected to have the average of the crystals effects $(5 \times 0.21 \%+0.42 \%) / 6=0.25 \%$ which agrees well with the analysis of the spectrum.

These data show the great advantage afforded by using a granular detector. If treated as one piece of germanium, the MARS detector would have the same amount of summing corrections as previous experiments which required detailed simulations and validation measurements. However, by separating the crystal into multiple smaller crystals, the individual crystal summing is reduced to at most $0.4 \%$ while still maintaining a high efficiency.

Table 8. Individual crystal summing corrections for MARS.

\begin{tabular}{|c|c|c|c|}
\hline Energy & Center crystal & Edge crystal & All front \\
\hline 1173 & $112395 \pm 343$ & $66984 \pm 266$ & $415522 \pm 667$ \\
\hline 1333 & $104577 \pm 325$ & $61160 \pm 250$ & $383037 \pm 626$ \\
\hline 2506 & $452 \pm 23$ & $136 \pm 15$ & $1016 \pm 40$ \\
\hline
\end{tabular}




\begin{tabular}{|l|l|l|l|}
\hline Summing & $(4.2 \pm 0.2) \times 10^{-3}$ & $(2.1 \pm 0.2) \times 10^{-3}$ & $(2.5 \pm 0.1) \times 10^{-3}$ \\
\hline
\end{tabular}

403

404

405

406

407

408

409

410

411

412

413

414

415

416

417

418

\section{9}

420

421

\subsubsection{Comparison of expected summing effect with measured}

For most of the experiment, the MARS detector was located as close to the target as possible, a distance estimated to be $7.45 \mathrm{~cm}$ based on external measurements and design drawings (see Section 2.3). The previous comparison used a relatively short run for the near measurement while this analysis used all of the on-resonance runs taken throughout the experiment. Summing effects were expected to be small compared to previous measurements because of the smaller crystal size in MARS and the larger distance to the target. To verify this, the detector was translated an additional $5 \mathrm{~cm}$ farther from the target and data were collected on resonance for $75 \mathrm{mC}$ of integrated beam current. Table 9 contains the results of that study and indicates a systematic downward shift in the ratios as the energy increases. It appears that geometric and attenuation effects are leading to a ratio that is not flat as a function of energy. This is supported by an analysis using the central crystal alone. While having smaller statistics, the central crystal should be less prone to geometric effects or effects caused by attenuation in intervening materials. Indeed, Table 10 shows that the counts per Coulomb for the central crystal is flat within the statistical uncertainties indicating that summing effects in a single crystal are indeed small.

Table 9. Comparison of near and far counts per Coulomb on resonance and the ratio.

\begin{tabular}{|l|l|l|l|}
\hline Energy $[\mathrm{keV}]$ & Near counts/C & Far counts/C & Near/Far \\
\hline 5183 & $28476 \pm 842$ & $14161 \pm 737$ & $2.01 \pm 0.12$ \\
\hline 6176 & $94791 \pm 2470$ & $53068 \pm 1636$ & $1.79 \pm 0.07$ \\
\hline 6793 & $34825 \pm 976$ & $21542 \pm 847$ & $1.62 \pm 0.08$ \\
\hline
\end{tabular}

Table 10. Comparison of near counts and far counts per Coulomb from central crystal only. The ratio is flat within the uncertainties.

\begin{tabular}{|c|c|c|c|}
\hline Energy $[\mathrm{keV}]$ & Near counts/C & Far counts/C (central crystal) & Near/Far \\
\hline 5183 & $28476 \pm 842$ & $3246 \pm 294$ & $8.77 \pm 0.84$ \\
\hline 6176 & $94791 \pm 2470$ & $12243 \pm 526$ & $7.74 \pm 0.39$ \\
\hline 6793 & $34825 \pm 976$ & $4140 \pm 284$ & $8.41 \pm 0.62$ \\
\hline
\end{tabular}


424

425

426

427

428

429

430

431

432

433

434

435

436

437

438

439

440

441

442

443

444

445

446

447

448

449

450

451

452

453

454

455

\section{Calculation of the Astrophysical S-factor}

\subsection{Extraction of Counts}

As mentioned earlier, a beam energy of $180 \mathrm{keV}\left(E_{\text {eff }}=163 \mathrm{keV}\right)$ was selected for the off-resonance runs. The counts in each peak were determined using the same background subtraction method as was used for the efficiency calculation. The regions of interest were determined for the on-resonance data and then used to extract the net counts for the off-resonance data. The counts in the full energy, first escape and second escape peaks were summed for each energy and are listed in Table 1.

Table 11. Net counts at each energy for $E_{\text {eff }}=163 \mathrm{keV}$. Counts are the sum of the full-energy, first-escape and second-escape peaks.

\begin{tabular}{|c|c|}
\hline Energy [keV] & Net Counts \\
\hline 5183 & $55 \pm 39$ \\
\hline 6176 & $132 \pm 31$ \\
\hline 6793 & $232 \pm 29$ \\
\hline GS & $32 \pm 27$ \\
\hline
\end{tabular}

\subsection{S-factor using efficiency}

Using the net number of events at each transition energy at $E_{\text {eff }}=163 \mathrm{keV}$ (Table 11), the efficiency at each energy (Table 4), and the accumulated charge of $79.49 \mathrm{C}$, the cross section, $\sigma(\mathrm{E})$, can be calculated using Equation (4.95) from Reference [16] :

$$
\sigma(\mathrm{E})=\frac{\epsilon_{\mathrm{eff}}}{\Delta \mathrm{E}_{\mathrm{tar}}} \frac{\mathrm{N}_{\gamma}}{\mathrm{N}_{\mathrm{p}}}[\mathrm{b}]
$$

where $\Delta \mathrm{E}_{\mathrm{tar}}$ is the thickness of the target in electronvolts $\left(10,000 \pm 1,000 \mathrm{eV}\right.$ for our targets), $\mathrm{N}_{\psi}$ is the number of detected counts in the peak corrected for efficiency (see Table 4), and $N_{p}$ is the number of incident protons. The astrophysical S-factor, $S(E)$ is then given by:

$$
S(E)=\frac{E_{c m}}{e^{-2 \pi \eta}} \sigma(E)
$$

where $E_{c m}$ is the center of mass energy of the interaction, and $\mathrm{e}^{-2 \pi n}$ is the Gamow factor where

$$
2 \pi \eta=0.989534 Z_{N} Z_{P} \sqrt{\frac{1}{E_{c m}} \frac{M_{N} M_{p}}{M_{N}+M_{p}}}
$$

The parameters from Table 3 were also used for the calculation. Since the efficiencies were determined using previous measurements, this approach is technically a relative measurement and one that goes through an intermediate stage thus potentially increasing the uncertainties. An alternate approach is described in the next subsection which reduces the uncertainties. The results of both methods will be compared there.

\section{$6.3 S$-factor from relative calculation}

In addition to the above approach, a second S-factor calculation was performed which was independent of the efficiency results thus reducing the uncertainties. This relative measurement is given by Equation (4.129) of Reference [16]: 
where the subscripts 1 and 2 refer to non-resonant and resonant, respectively. In addition, $B, N, \eta$, and $W$ are the branching ratio, total number of detected particles or photons, detector efficiency, and angular correlation, respectively. The efficiency term $\frac{N_{p, 2}}{N_{p, 1}\left(E_{0,1}\right)}$ cancels out as the secondary $\gamma$-ray occurs at the same energy for both on- and off-resonance runs. It is also assumed that there is no angular correlation, so that $\frac{W_{2}}{W_{1}\left(E_{0,1}\right)}=1$. It is further assumed that there is $100 \%$ feeding from the primary to the secondary transition so that $B_{1}\left(E_{0,1}\right)=1$. The $N_{i}$ and $N_{p, i}$ terms are the number of detected $\gamma$-rays detected and number of incidence protons, respectively. The stopping powers, $\varepsilon$, are taken from

467 SRIM[12]; $\lambda_{\mathrm{dB}}$ is the deBroglie wavelength; $\Delta \mathrm{E}_{1}\left(\mathrm{E}_{0,1}\right)$ is the target thickness; and $\omega \gamma_{2}$ is the resonance 468 strength. Figure 11 shows the S-factor results along with those from LENA [7] and LUNA [4-6]. Table 12 compares the two approaches for calculating the cross-section. As would be expected, the relative approach reduces the uncertainty (and also happens to increases the central values). The key item to note is that this measurement yielded the same S-factors as the previous measurement and with comparable uncertainties. The new measurement was made in less time (one week versus one month) and had considerably smaller systematic uncertainties than the previous measurement at LENA owing to 474 the lack of large summing corrections. However, with the small reduction in uncertainty and only this 475 one case, strong conclusions should not be drawn about the relative merits of the methods.

476 Table 12. Comparison of S-factors for the strongest transitions to compare the two calculational approaches.

\begin{tabular}{|c|c|c|}
\hline Transition [keV] & S-factor using efficiency & S-factor relative measurement \\
\hline 6176 & $0.642 \pm 0.171(26.6 \%)$ & $0.695 \pm 0.181(26.0 \%)$ \\
\hline 6793 & $1.212 \pm 0.214(17.7 \%)$ & $1.310 \pm 0.221(16.9 \%)$ \\
\hline
\end{tabular}



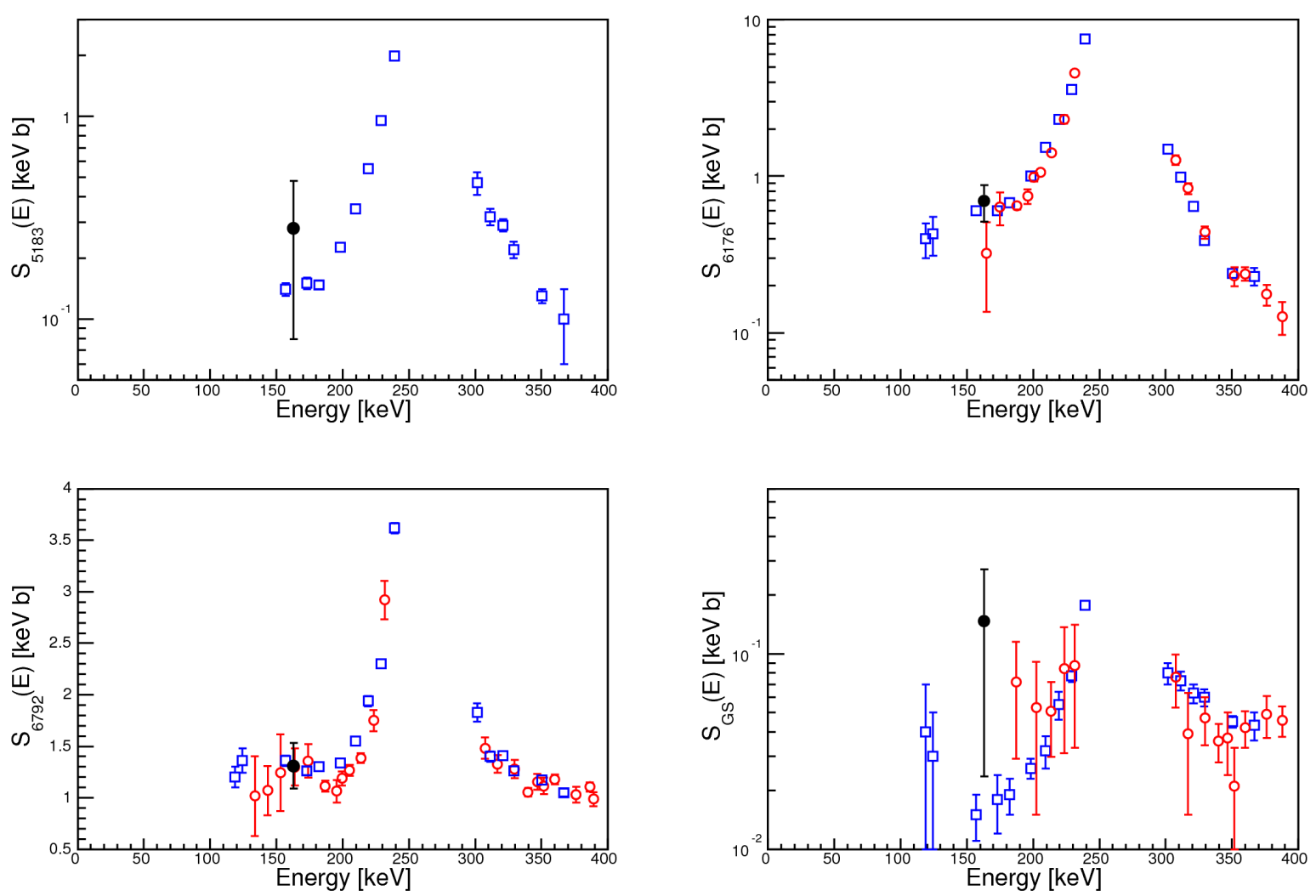

479

480

481

Figure 11. S-factors for MARS, LENA and LUNA published results. Black dots: Current results LENA-MARS, Red open circles: LENA-Runkle[7], Blue open squares: LUNA[4-6].

\section{Conclusions}

The MARS detector was successfully transported to LENA and was used to measure the S-factor for the ${ }^{14} \mathrm{~N}(\mathrm{p}, \gamma)^{15} \mathrm{O}^{*}$ reaction at $\mathrm{E}_{\text {eff }}=163 \mathrm{keV}$. The granular nature of the detector reduced the summing corrections considerably and the tolerance of higher rates allowed a measurement with precision similar to previous attempts to be performed in less time than previously. The detector was stable during the measurement and all of the efficiency and summing cross checks agreed with expectations. The ultimate precision for the $\mathrm{E}_{\text {eff }}=163 \mathrm{keV}$ data point was limited by the time the detector was available and a longer run would reduce the statistical uncertainties further. In addition, there would be some advantage to redesigning the front window of the MARS detector so that the target can be brought even closer to the front face of the crystals. As mentioned previously, the MARS detector was originally designed for stand-off detection where the source distances are typically tens of meters. In this experiment, every centimeter closer to the target will save time through increased efficiency. This would then allow the measurement of the S-factor down to lower energies and increase the precision at higher energies both of which will act to reduce the uncertainties when extrapolated down to astrophysically relevant energies. 


\section{Acknowledgements}

This document is PNNL-SA-98562. A portion of the research described in this paper is part of the Ultra-sensitive Nuclear Measurements Initiative at Pacific Northwest National Laboratory. It was conducted under the Laboratory Directed Research and Development program at PNNL, a multiprogram national laboratory operated by Battelle for the U.S. Department of Energy under contract DE-AC0576RL01830. The MARS detector was funded by the Office of Defense Nuclear Nonproliferation, Office of Nonproliferation Research and Development (NA-22) and the authors thank the Office for allowing the detector to be used in this work.

\section{References}

[1] J.E. Fast, C.E. Aalseth, D.M. Asner, C.A. Bonebrake, A.R. Day, K.E. Dorow, E.S. Fuller, B.D. Glasgow, T.W. Hossbach, B.J. Hyronimus, J.L. Jensen, K.I. Johnson, D.V. Jordan, G.P. Morgen, S.J. Morris, O.D. Mullen, A.W. Myers, W.K. Pitts, J.S. Rohrer, R.C. Runkle, A. Seifert, J.M. Shergur, S.C. Stave, G. Tatishvili, R.C. Thompson, L.C. Todd, G.A. Warren, J.A. Willett, L.S. Wood, The Multi-sensor Airborne Radiation Survey (MARS) instrument, Nuclear Instruments and Methods in Physics Research Section A: Accelerators, Spectrometers, Detectors and Associated Equipment, 698 (2013) 152-167.

[2] J.M. Cesaratto, A.E. Champagne, T.B. Clegg, M.Q. Buckner, R.C. Runkle, A. Stefan, Nuclear astrophysics studies at LENA: The accelerators, Nuclear Instruments \& Methods in Physics Research Section a-Accelerators Spectrometers Detectors and Associated Equipment, 623 (2010) 888-894.

[3] R.J. Gehrke, R.G. Helmer, R.C. Greenwood, Precise Relative Gamma-Ray Intensities for Calibration of Ge Semiconductor-Detectors, Nucl Instrum Methods, 147 (1977) 405-423.

[4] M. Marta, A. Formicola, D. Bemmerer, C. Broggini, A. Caciolli, P. Corvisiero, H. Costantini, Z. Elekes, Z. Fulop, G. Gervino, A. Guglielmetti, C. Gustavino, G. Gyurky, G. Imbriani, M. Junker, A. Lemut, B. Limata, C. Mazzocchi, R. Menegazzo, P. Prati, V. Roca, C. Rolfs, C.R. Alvarez, E. Somorjai, O. Straniero, F. Strieder, F. Terrasi, H.P. Trautvetter, A. Vomiero, L. Collaboration, The N-14(p,gamma)O-15 reaction studied with a composite germanium detector, Physical Review C, 83 (2011) 045804.

[5] M. Marta, E. Trompler, D. Bemmerer, R. Beyer, C. Broggini, A. Caciolli, M. Erhard, Z. Fulop, E. Grosse, G. Gyurky, R. Hannaske, A.R. Junghans, R. Menegazzo, C. Nair, R. Schwengner, T. Szucs, S. Vezzu, A. Wagner, D. Yakorev, Resonance strengths in the N-14(p,gamma)O-15 and N-15(p,alpha gamma)C-12 reactions, Physical Review C, 81 (2010) 055807.

[6] A. Lemut, D. Bemmerer, F. Confortola, R. Bonetti, C. Broggini, P. Corvisiero, H. Costantini, J. Cruz, A. Formicola, Z. Fulop, G. Gervino, A. Guglielmetti, C. Gustavino, G. Gyurky, G. Imbriani, A.P. Jesus, M. Junker, B. Limata, R. Menegazzo, P. Prati, V. Roca, D. Rogalla, C. Rolfs, M. Romano, C.R. Alvarez, F. Schumann, E. Somorjai, O. Straniero, F. Strieder, F. Terrasi, H.P. Trautvetter, First measurement of the N14(p, gamma)O-15 cross section down to 70 keV, Physics Letters B, 634 (2006) 483-487.

[7] R.C. Runkle, A.E. Champagne, C. Angulo, C. Fox, C. Iliadis, R. Longland, J. Pollanen, Direct measurement of the N-14(p,gamma)O-15 S factor, Physical Review Letters, 94 (2005) 082503.

[8] D.A. Vermilyea, THE KINETICS OF FORMATION AND STRUCTURE OF ANODIC OXIDE FILMS ON TANTALUM, Acta Metallurgica, 1 (1953) 282. 
[9] P.J. Attayek, E.S. Meyer, L. Lina, G.C. Rich, T.B. Clegg, O. Coronell, A remotely controlled, semiautomatic target system for Rutherford backscattering spectrometry and elastic recoil detection analyses of polymeric membrane samples, Nuclear Instruments \& Methods in Physics Research Section a-Accelerators Spectrometers Detectors and Associated Equipment, 676 (2012) 21-25.

[10] J. Keinonen, A. Anttila, PREPARATION OF IMPLANTED NITROGEN TARGETS, Nuclear Instruments \& Methods, 160 (1979) 211-215.

[11] M. Riihonen, J. Keinonen, MEASUREMENTS OF ABSOLUTE RESONANCE STRENGTHS IN (P,GAMMA) REACTIONS ON RARE OR GASEOUS NUCLEI, Nuclear Instruments \& Methods, 144 (1977) 323-329.

[12] J.F. Ziegler, J.P. Biersack, M.D. Ziegler, SRIM, the stopping and range of ions in matter, SRIM Co., Chester, Maryland, 2008.

[13] G.F. Knoll, Radiation Detection and Measurements (fourth edition), John Wiley and Sons, New York, 2010, pp. 830.

[14] P. Sangsingkeow, K.D. Berry, E.J. Dumas, T.W. Raudorf, T.A. Underwood, Advances in germanium detector technology, Nuclear Instruments and Methods in Physics Research Section A: Accelerators, Spectrometers, Detectors and Associated Equipment, 505 (2003) 183-186.

[15] W. Hennig, Y.X. Chu, H. Tan, A. Fallu-Labruyere, W.K. Warburton, The DGF pixie-4 spectrometer compact digital readout electronics for HPGe clover detectors, Nucl Instrum Meth B, 263 (2007) 175178.

[16] C. Iliadis, Nuclear Physics of Stars, Wiley-VCH Verlag GmbH, Weinheim, FRG, 2008.

[17] E.G. Adelberger, A. Garcia, R.G.H. Robertson, K.A. Snover, A.B. Balantekin, K. Heeger, M.J. RamseyMusolf, D. Bemmerer, A. Junghans, C.A. Bertulani, J.W. Chen, H. Costantini, P. Prati, M. Couder, E. Uberseder, M. Wiescher, R. Cyburt, B. Davids, S.J. Freedman, M. Gai, D. Gazit, L. Gialanella, G. Imbriani, U. Greife, M. Hass, W.C. Haxton, T. Itahashi, K. Kubodera, K. Langanke, D. Leitner, M. Leitner, P. Vetter, L. Winslow, L.E. Marcucci, T. Motobayashi, A. Mukhamedzhanov, R.E. Tribble, K.M. Nollett, F.M. Nunes, T.S. Park, P.D. Parker, R. Schiavilla, E.C. Simpson, C. Spitaleri, F. Strieder, H.P. Trautvetter, K. Suemmerer, S. Typel, Solar fusion cross sections. II. The pp chain and CNO cycles, Rev Mod Phys, 83 (2011) 195-245.

[18] J. Beringer, J.F. Arguin, R.M. Barnett, K. Copic, O. Dahl, D.E. Groom, C.J. Lin, J. Lys, H. Murayama, C.G. Wohl, W.M. Yao, P.A. Zyla, C. Amsler, M. Antonelli, D.M. Asner, H. Baer, H.R. Band, T. Basaglia, C.W. Bauer, J.J. Beatty, V.I. Belousov, E. Bergren, G. Bernardi, W. Bertl, S. Bethke, H. Bichsel, O. Biebel, E. Blucher, S. Blusk, G. Brooijmans, O. Buchmueller, R.N. Cahn, M. Carena, A. Ceccucci, D. Chakraborty, M.C. Chen, R.S. Chivukula, G. Cowan, G. D'Ambrosio, T. Damour, D. de Florian, A. de Gouvea, T. DeGrand, P. de Jong, G. Dissertori, B. Dobrescu, M. Doser, M. Drees, D.A. Edwards, S. Eidelman, J. Erler, V.V. Ezhela, W. Fetscher, B.D. Fields, B. Foster, T.K. Gaisser, L. Garren, H.J. Gerber, G. Gerbier, T. Gherghetta, S. Golwala, M. Goodman, C. Grab, A.V. Gritsan, J.F. Grivaz, M. Grunewald, A. Gurtu, T. Gutsche, H.E. Haber, K. Hagiwara, C. Hagmann, C. Hanhart, S. Hashimoto, K.G. Hayes, M. Heffner, B. Heltsley, J.J. Hernandez-Rey, K. Hikasa, A. Hocker, J. Holder, A. Holtkamp, J. Huston, J.D. Jackson, K.F. Johnson, T. Junk, D. Karlen, D. Kirkby, S.R. Klein, E. Klempt, R.V. Kowalewski, F. Krauss, M. Kreps, B. Krusche, Y.V. Kuyanov, Y. Kwon, O. Lahav, J. Laiho, P. Langacker, A. Liddle, Z. Ligeti, T.M. Liss, L. Littenberg, K.S. Lugovsky, S.B. Lugovsky, T. Mannel, A.V. Manohar, W.J. Marciano, A.D. Martin, A. Masoni, J. Matthews, D. Milstead, R. Miquel, K. Monig, F. Moortgat, K. Nakamura, M. Narain, P. Nason, 
S. Navas, M. Neubert, P. Nevski, Y. Nir, K.A. Olive, L. Pape, J. Parsons, C. Patrignani, J.A. Peacock, S.T. Petcov, A. Piepke, A. Pomarol, G. Punzi, A. Quadt, S. Raby, G. Raffelt, B.N. Ratcliff, P. Richardson, S. Roesler, S. Rolli, A. Romaniouk, L.J. Rosenberg, J.L. Rosner, C.T. Sachrajda, Y. Sakai, G.P. Salam, S. Sarkar, F. Sauli, O. Schneider, K. Scholberg, D. Scott, W.G. Seligman, M.H. Shaevitz, S.R. Sharpe, M. Silari, T. Sjostrand, P. Skands, J.G. Smith, G.F. Smoot, S. Spanier, H. Spieler, A. Stahl, T. Stanev, S.L. Stone, T. Sumiyoshi, M.J. Syphers, F. Takahashi, M. Tanabashi, J. Terning, M. Titov, N.P. Tkachenko, N.A. Tornqvist, D. Tovey, G. Valencia, K. van Bibber, G. Venanzoni, M.G. Vincter, P. Vogel, A. Vogt, W. Walkowiak, C.W. Walter, D.R. Ward, T. Watari, G. Weiglein, E.J. Weinberg, L.R. Wiencke, L. Wolfenstein, J. Womersley, C.L. Woody, R.L. Workman, A. Yamamoto, G.P. Zeller, O.V. Zenin, J. Zhang, R.Y. Zhu, P.D. Grp, Review of Particle Physics Particle Data Group, Phys Rev D, 86 (2012) 1.

[19] G. Audi, A.H. Wapstra, C. Thibault, The AME2003 atomic mass evaluation (II). Tables, graphs and references, Nucl Phys A, 729 (2003) 337-676.

[20] I.J. Kim, C.S. Park, H.D. Choi, Absolute calibration of Co-60 by using sum-peak method and an HPGe detector, Appl Radiat Isotopes, 58 (2003) 227-233.

\footnotetext{
${ }^{1}$ XIA LLC, Hayward, CA, http://xia.com
} 